\title{
Letter
}

\section{Chaos Suppression of a Class of Fractional-Order Chaotic Systems with Order Lying in $(1,2)$}

\author{
Kang Xu \\ Anhui Huitong Space Geographic Information Technology Co., Ltd, Hefei, China
}

Email address:

xukang1984@aliyun.com

\section{To cite this article:}

Kang Xu. Chaos Suppression of a Class of Fractional-Order Chaotic Systems with Order Lying in (1, 2). Mathematics Letters. Vol. 4, No. 3, 2018, pp. 51-58. doi: 10.11648/j.ml.20180403.13

Received: September 25, 2018; Accepted: October 30, 2018; Published: December 4, 2018

\begin{abstract}
It is shown that fractional-order (FO) nonlinear systems can also show higher nonlinearity and complex dynamics. FO chaotic systems have wider applications in secure communication, signal processing, financial field due to FO chaos has larger key space and more complex random sequences than integer-order chaos. Thanks to the lack of the effective analytical methods and controller design methods of integer-order chaotic systems can not be applied directly to FO chaos systems, to control chaos of FO chaotic systems is a very interesting and difficult problem, especially for FO chaotic system with order $\alpha: 1<\alpha<2$. Based on the stability theory of FO systems and the linear state feedback control, an LMI criterion for controlling a class of fractional-order chaotic systems with fractional-order $\alpha: 1<\alpha<2$ is addressed in this paper. The proposed method can be easily verified and resolved by using the Matlab LMI toolbox. Moreover, the proposed controller is linear, easy to implement and overcome some defects in the recent literature, which have improved the existing results. The method employed in this letter can effectively avoid control cost and inaccuracy in the literatures, and can be be applied to FO hyperchaos systems and synchronization controller design of FO chaotic system. Theoretical analysis and numerical simulations are presented to demonstrate the validity and feasibility of the proposed methods.
\end{abstract}

Keywords: Fractional-Order Chaotic Systems, Linear Control, Linear Matrix Inequality

\section{Introduction}

The main property of chaotic dynamics is its critical sensitivity to initial conditions, which is responsible for initially neighboring trajectories separating from each other exponentially in the course of time. This feature would lead systems to unstable, performance-degraded, and even catastrophic situations. Therefore, it is considered as undesirable and should be eliminated in many cases. Due to its potential applications in various branches of engineering such as mechanical systems, electrical and electronic systems, chaos control has attracted increasing attention in recent years [1-4]. Many researchers and engineers have devoted their efforts to chaos control, including stabilization of unstable equilibrium and controlling the chaotic states to desired states. Some methods have been applied to deal with this problem, such as OGY method [5], the feedback control method [6], impulsive control [7], backstepping method [8], adaptive control [9], bang-bang control [10], sliding mode control [11], nonlinear control [12], active control [13], and many others. On the other hand, during the past several years, with the development of theory of fractional-order calculus, it has been surprisingly found that many FO differential systems also display complex bifurcation and chaos phenomena, for instance, FO Duffing's oscillators [14], FO Chua's circuit system [15], FO Rössler system [16], FO Chen system [17], FO Lorenz system [18], FO Lü system [19], FO Liu system [20], FO financial system [21], FO Volta's system [22], FO hyperchaotic Chen system [23], FO hyperchaotic Lorenz system [24], FO hyperchaotic Rössler system [25], FO love model [26] and so on. Naturally, to control chaos in FO chaotic system has become a hot issue.

Note that the proposed schemes in most of the previous works about chaos control, are too complex in design to implement in applications, meanwhile, some of these control schemes are not applicable to fractional-order chaotic systems. Compared with nonlinear control methods, due to the 
simplicity in configuration and implementation, the linear feedback control is especially attractive and has been commonly adopted for practical implementations. It possesses a high value in application. On the other hand, most of the above mentioned works on chaos control all are dependent on the Lyapunov direct method. Due to the difference between fractional-order differential systems and integer-order differential ones, it is very difficult and inconvenient to construct Lyapunov functions for fractional-order systems. Some authors used linearization approach to achieve chaos control based on linear control [27-30], however, inaccuracy may be encountered.

In this paper, the aim of the paper is to control chaos in fractional-order systems by using the economic, simply implemented control scheme and avoid error caused by linearization in many present literatures. Based on the stability theory of fractional-order differential system and the linear feedback control, chaos control of a class of chaotic fractional-order systems is considered, a LMI conditions is established, feedback gains could be found directly from the LMI formulation. It would be specially mentioned that control of FO system with order $(1,2)$ is discussed. To the best of our knowledge, there are few results on the issue. Finally, a numerical example is given to illustrate the effectiveness and usefulness of the proposed method.
The remainder of this brief is organized as follows. In Section 2, problem description and some preliminary results are presented. In Section 3, main results are derived. Example and corresponding numerical simulation are shown in Section 4. Finally, conclusions are drawn in Section 5.

Notations: Throughout this paper, $\mathrm{R}^{\mathrm{n}}$ and $\mathrm{R}^{\mathrm{n} \times \mathrm{m}}$, denote, respectively, the $\mathrm{n}$-dimensional Euclidean space and the set of all $\mathrm{n} \times \mathrm{m}$ real matrices; $\mathrm{M}^{\mathrm{T}}$ denotes transpose of matrix $\mathrm{M}$; The notation $\mathrm{M}>0(M<0)$ means that the matrix $\mathrm{M}$ is positive (negative) definite; $\operatorname{Sym}\{M\}$ is used to denote the expression $\mathrm{M}+\mathrm{M}^{\mathrm{T}}$, and $\star$ is used to denote a block matrix element that is induced by transposition.

\section{Problem Description and Preliminaries}

The fractional-order deals with derivatives and integrals to an arbitrary order (real or, even, complex order). Note that the initial conditions of Caputo derivative differential equations with Caputo derivatives take on the same form as for integer-order differential, which have well understood physical meanings and more applications in modelling and analysis. Here, Caputo derivative is taken.

Definition 1. The Caputo derivative of fractional order $\alpha$ of function $\mathrm{x}(\mathrm{t})$ is defined as follows:

$$
D_{t_{0}, t}^{\alpha} x(t)=D_{t_{0}, t}^{-(n-\alpha)} \frac{d^{n}}{d t^{n}} x(t)=\frac{1}{\Gamma(n-\alpha)} \int_{t_{0}}^{t}(t-\tau)^{(n-\alpha-1)} x^{(n)}(\tau) d \tau,
$$

where $D_{t_{0}, t}^{-\alpha} x(t)=\frac{1}{\Gamma(\alpha)} \int_{t_{0}}^{t}(t-\tau)^{\alpha-1}(n)(\tau) d \tau, \quad n-1 \leq \alpha<n \in Z^{+}$.

$\Gamma(\cdot)$ is the gamma function, $\Gamma(\tau)=\int_{0}^{\infty} t^{\tau-1} e^{-t} d t$.

Consider the following fractional-order chaotic systems

$$
D^{\alpha} x=f(x)=\left(A_{0}+\Delta A(x)\right) x,
$$

where $x=\left(x_{1}, x_{2} \cdots, x_{n}\right)^{T} \in R^{n}$ denotes state vectors of the state system, $f: R^{n} \rightarrow R^{n}$ define nonlinear vector field in n-dimensional vector space, $A_{0} \in R^{n \times n}$ is a real coefficient matrix of the linear part of system (1), $\Delta A(x) \in R^{n \times n}$ is a matrix state variable, order $\alpha: 1<\alpha<2$.

Corresponding linear controlled fractional-order system is given by

$$
D^{\alpha} x=\left(A_{0}+\Delta A(x)+K\right) x,
$$

It can be seen from controlled system (2) that $\Delta A(x) \in R^{n \times n}$ is a matrix, and every element of the matrix is bounded for the boundedness of chaotic systems, thus, $\Delta A(x)$ is regarded as a bounded perturbation item of coefficient matrix $A_{0}$ of system (2) and is of form: $\Delta A(x)=A_{M}\left[\delta_{i j}\right]=\left[\gamma_{i j}\right]_{n \times n}\left[\delta_{i j}\right]_{n \times n} \quad, \quad$ where $\quad \gamma_{i j} \geq 0$, $-1 \leq \delta_{i j} \leq 1, \quad A_{M}=\left[\gamma_{i j}\right]_{n \times n}$ is the maximal perturbation scope matrix. In order to deal with the perturbation $\Delta A(x)$, based on method presented in [31], controlled system (2) could be rewritten as follows

$$
D^{\alpha} x=\left(A_{0}+K+D F E\right) x,
$$

where

$$
D=\left[\sqrt{\gamma_{11}} e_{1}^{n}, \cdots, \sqrt{\gamma_{1 n}} e_{1}^{n}, \cdots, \sqrt{\gamma_{n 1}} e_{1}^{n}, \cdots, \sqrt{\gamma_{n n}} e_{n}^{n}\right]_{n \times n^{2}},
$$

where Kx denotes linear state feedback controller, $K \in R^{n \times n}$ is the feedback gains.

$$
F \in H=\left\{\operatorname{diag}\left[\delta_{11}, \cdots, \quad \delta_{1 n}, \cdots, \delta_{n 1}, \cdots, \quad \delta_{n n}, \cdots\right] \in R^{n^{2} \times n^{2}},\left|\delta_{i j}\right| \leq 1\right\},
$$

$$
E=\left[\sqrt{\gamma_{11}} e_{1}^{n}, \cdots, \sqrt{\gamma_{1 n}} e_{1}^{n}, \cdots, \sqrt{\gamma_{n 1}} e_{1}^{n}, \cdots, \sqrt{\gamma_{n n}} e_{n}^{n}\right]_{n^{2} \times n},
$$

$e_{k}^{n} \in R^{n}$ denote the column vectors with the k-th element being 1 and all the others being 0 .

The aim of this work is to design a suitable feedback gain matrix $\mathrm{K}$ such that controlled dynamical system (3) is asymptotically stable. To this end, the following lemmas need 
to be introduced.

Lemma 1 [32]. For fractional-order linear system with order $\alpha: D^{\alpha} x=A x$ is asymptotically stable if and only if

$$
|\arg (\operatorname{spec}(A))|>\frac{\alpha \pi}{2}
$$

where $A \in R^{n \times n}$ is a deterministic real matrix, spec (A) is the spectrum of $\mathrm{A}$.

Lemma 2 [33]. Let $A \in R^{n \times n}$ be a real matrix. Then $|\arg (\operatorname{spec}(A))|>\frac{\alpha \pi}{2}$, where, $1 \leq \alpha<2$ if and only if there exists $P>0$ such that

$$
\left[\begin{array}{cl}
\left(A P+P A^{T}\right) \sin \theta & \left(A P-P A^{T}\right) \cos \theta \\
* & \left(A P+P A^{T}\right) \sin \theta
\end{array}\right]<0,
$$

Where $\theta=\pi-\alpha \pi / 2$.

Lemma 3 [34]. Let $X$ and $Y$ be matrices of appropriate dimensions, then the following inequality holds for $\forall \varepsilon>0$,

$$
X^{T} Y+Y^{T} X \leq \varepsilon X^{T} X+\varepsilon^{-1} Y^{T} Y .
$$

Lemma 4 [35] (Schur complement). For a real matrix $M=M^{T}$, the following conditions are equivalent:

$$
\begin{gathered}
(i) M=\left[\begin{array}{cc}
M_{11} & M_{12} \\
* & M_{22}
\end{array}\right]<0, \\
(\text { ii }) M_{11}<0, M_{22}-M_{12}^{T} M_{11}^{-1} M_{12}>0, \\
\text { (iii) } M_{22}<0, M_{11}-M_{12} M_{22}^{-1} M_{12}^{T}>0 .
\end{gathered}
$$

Remark 1. Many fractional-order chaotic systems could be expressed by (1). Examples include FO Chen system [17], FO Lorenz system [18], FO Lü system [19], FO Liu system [20],
FO financial system [21], FO Volta's system [22], FO hyperchaotic Chen system [23], FO hyperchaotic Lorenz system [24] and so on.

\section{Main Results}

In this section, a LMI conditions is presented for chaos control in fractional-order chaotic systems with fractional-order $\alpha: 1<\alpha<2$.

Theorem 1. Controlled system (2) with fractional-order $\alpha(1<\alpha<2)$ is asymptotically stable if there exists a symmetric positive definite matrix $P$, a matrix $X$, and a real scalar $\varepsilon>0$, such that

$$
\left[\begin{array}{cccc}
\Omega_{11} & \Omega_{12} & P E^{T} & 0 \\
* & \Omega_{22} & 0 & P E^{T} \\
* & * & -\varepsilon I & 0 \\
* & * & * & -\varepsilon I
\end{array}\right]<0,
$$

where

$$
\begin{gathered}
\Omega_{11}=\Omega_{22}=\left(A_{0} P+P A_{0}^{T}+X+X^{T}\right) \sin \theta+\varepsilon D D^{T}, \\
\Omega_{12}=\left(A_{0} P-P A_{0}^{T}+X-X^{T}\right) \cos \theta, \\
\theta=\pi-\alpha \pi / 2 .
\end{gathered}
$$

Moreover, feedback gain matrix is given by

$$
K=X P^{-1} \text {. }
$$

Proof. Let $A=A_{0}+K+\Delta A(x)$, suppose that there exist symmetrical matrix $P>0$, a matrix $X$, and scalars $\varepsilon>0$ such that (8) and (9) hold, it follows that

$$
\begin{aligned}
& {\left[\begin{array}{cc}
\left(A P+P A^{T}\right) \sin \theta & \left(A P-P A^{T}\right) \cos \theta \\
* & \left(A P+P A^{T}\right) \sin \theta
\end{array}\right]} \\
& =\left[\begin{array}{cc}
\left(A_{0} P+P A_{0}^{T}+X+X^{T}\right) \sin \theta & \left(A_{0} P-P A_{0}^{T}+X-X^{T}\right) \cos \theta \\
\left(A_{0} P-P A_{0}^{T}+X-X^{T}\right) \cos \theta & \left(A_{0} P+P A_{0}^{T}+X+X^{T}\right) \sin \theta
\end{array}\right]+S y m\left\{\left[\begin{array}{cc}
D \sin \theta & D \cos \theta \\
-D \cos \theta & D \sin \theta
\end{array}\right]\left[\begin{array}{cc}
F & 0 \\
0 & F
\end{array}\right]\left[\begin{array}{cc}
E P & 0 \\
0 & E P
\end{array}\right]\right\} .
\end{aligned}
$$

By using Lemma 3, the following inequality holds for $\forall \varepsilon>0$

$$
\begin{aligned}
& \operatorname{Sym}\left\{\left[\begin{array}{cc}
D \sin \theta & D \cos \theta \\
-D \cos \theta & D \sin \theta
\end{array}\right]\left[\begin{array}{cc}
F & 0 \\
0 & F
\end{array}\right]\left[\begin{array}{cc}
E P & 0 \\
0 & E P
\end{array}\right]\right\} \\
& \leq \varepsilon\left[\begin{array}{cc}
D \sin \theta & D \cos \theta \\
-D \cos \theta & D \sin \theta
\end{array}\right]\left[\begin{array}{cc}
D \sin \theta & D \cos \theta \\
-D \cos \theta & D \sin \theta
\end{array}\right]^{T}+\varepsilon^{-1}\left[\begin{array}{cc}
E P & 0 \\
0 & E P
\end{array}\right]^{T}\left[\begin{array}{cc}
E P & 0 \\
0 & E P
\end{array}\right] .
\end{aligned}
$$

Substituting (11) into (10), one obtains 


$$
\begin{aligned}
& {\left[\begin{array}{ll}
\left(A P+P A^{T}\right) \sin \theta & \left(A P-P A^{T}\right) \cos \theta \\
\left(P A^{T}-A P\right) \cos \theta & \left(A P+P A^{T}\right) \sin \theta
\end{array}\right]} \\
& =\left[\begin{array}{ll}
\left(A_{0} P+P A_{0}^{T}+X+X^{T}\right) \sin \theta & \left(A_{0} P-P A_{0}^{T}+X-X^{T}\right) \cos \theta \\
\left(A_{0} P-P A_{0}^{T}+X-X^{T}\right) \cos \theta & \left(A_{0} P+P A_{0}^{T}+X+X^{T}\right) \sin \theta
\end{array}\right]+\varepsilon\left[\begin{array}{cc}
D \sin \theta & D \cos \theta \\
-D \cos \theta & D \sin \theta
\end{array}\right]\left[\begin{array}{cc}
D \sin \theta & D \cos \theta \\
-D \cos \theta & D \sin \theta
\end{array}\right]^{T}+\varepsilon^{-1}\left[\begin{array}{cc}
E P & 0 \\
0 & E P
\end{array}\right]^{T}\left[\begin{array}{cc}
E P & 0 \\
0 & E P
\end{array}\right] \\
& =\left[\begin{array}{cc}
\Omega_{11} & \Omega_{12} \\
* & \Omega_{22}
\end{array}\right]+\varepsilon^{-1}\left[\begin{array}{cc}
E P & 0 \\
0 & E P
\end{array}\right]^{T}\left[\begin{array}{cc}
E P & 0 \\
0 & E P
\end{array}\right] .
\end{aligned}
$$

By applying Lemma 4 (Schur complement) to (8) and combining with (12), it holds that

$$
\left[\begin{array}{ll}
\left(A P+P A^{T}\right) \sin \theta & \left(A P-P A^{T}\right) \cos \theta \\
\left(P A^{T}-A P\right) \cos \theta & \left(A P+P A^{T}\right) \sin \theta
\end{array}\right]<0 .
$$

It follows from Lemma 2 and (13) that controlled system (2) with the feedback gain (9) is asymptotically stable.

Remark 2. Note that most of the existing results about control of fractional order systems are based on Lemma 1. Refs. [11-13, 36] adopted such as sliding control, active control, and nonlinear feedback control such that eigenvalues of the coefficient matrix of controlled systems are in a certain range, thereby controlling chaos in fractional-order systems. Although these methods are able to achieve better effect, control cost will be high and it is hard to implement. Refs. [27-30] attempted to overcome these shortages by using linear control. However, due to the coefficient matrix of nonlinear system usually contain system variables, it is not very easy to obtain eigenvalues of the coefficient matrix, therefore, they all used linearization approach to achieve such a goal, which may make inaccuracy in this instance. The method employed in this letter can effectively avoid control cost and inaccuracy.

Remark 3. Linear matrix inequality (LMI) approach is an popular method to deal with stabilization of integer-order systems, but it has to rely more on constructing the suitable Lyapunov function, which has no application to fractional-order systems. There are few papers to consider stabilization of chaotic fractional-order systems by using LMI technique. It must be noted that the advantage of this approach is to obtain directly feedback gains from the LMI formulation in the MATLAB environment, which also improves the existing results.

Remark 4. Some of the results about chaos control of fractional-order chaotic system reported in literatures all concentrated on the case of fractional-order $\alpha: 0<\alpha<1$, the case of fractional-order $\alpha: 1<\alpha<2$ is still given less concern.

\section{Numerical Example}

In this section, fractional-order chaotic Lorenz system with fractional-order $\alpha=1.05$ is taken as an example to verify and demonstrate the effectiveness of the proposed method.

Fractional-order chaotic Lorenz system [18] is described by

$$
\left\{\begin{array}{c}
D^{\alpha} x_{1}=a\left(x_{2}-x_{1}\right) \\
D^{\alpha} x_{2}=b x_{1}-x_{1} x_{3}-x_{2} \\
D^{\alpha} x_{3}=x_{1} x_{2}-c x_{3}
\end{array}\right.
$$

when the parameters are taken as $a=10, b=28, c=8 / 3$ and $\alpha=1.05$, system (14) exhibits chaotic behaviour as shown in Figure 1.

The controlled system is defined as follows

$$
D^{\alpha} x=\left(A_{0}+\Delta A(x)+K\right) x
$$

where

$$
A_{0}=\left[\begin{array}{ccc}
-a & a & 0 \\
b & -1 & 0 \\
0 & 0 & -c
\end{array}\right], \Delta A(x)=\left[\begin{array}{ccc}
0 & 0 & 0 \\
0 & 0 & -x_{1} \\
x_{2} & 0 & 0
\end{array}\right]
$$

Based on (3) and the boundedness of chaotic system, one obtains

$$
D=\left[\begin{array}{ccc}
000000 & 0 & 00 \\
000005 & 0 & 00 \\
000000 & \sqrt{30} & 00
\end{array}\right], E=\left[\begin{array}{cccc}
000000 \sqrt{30} & 00 \\
000000 & 0 & 00 \\
000005 & 0 & 00
\end{array}\right]^{T} .
$$

Thus, by using Theorem 2 and the Matlab's LMI toolbox, the feasible solution of (8) is given by

$$
\begin{gathered}
P=\left[\begin{array}{ccc}
88.0297 & 3.7848 & -4.8671 \\
3.7848 & 252.1759 & -1.4968 \\
-4.8671 & -1.4968 & 84.5999
\end{array}\right], \\
X=10^{3} \times\left[\begin{array}{ccc}
0.5557 & -3.2111 & -0.0767 \\
-3.2111 & -4.9989 & 0.0412 \\
-0.0767 & 0.0412 & -6.0018
\end{array}\right], \\
\varepsilon=795.3859 .
\end{gathered}
$$

Based on (9), feedback gain $\mathrm{K}$ is given as follows

$$
K=X P^{-1}=\left[\begin{array}{ccc}
6.8236 & -12.8404 & -0.7416 \\
-35.7535 & -19.2978 & -1.9111 \\
-4.8015 & -0.1872 & -71.2234
\end{array}\right]
$$

In the numerical simulations, the initial states of the controlled systems is taken as $x_{1}(0)=4, x_{2}(0)=2, x_{3}(0)=11$. Substituting 
the feedback gain $K$ into the controlled system (14). Figure 2. the linear control law. Figure 3 describes the control input. shows that the Lorenz system can be stabilized to the origin with
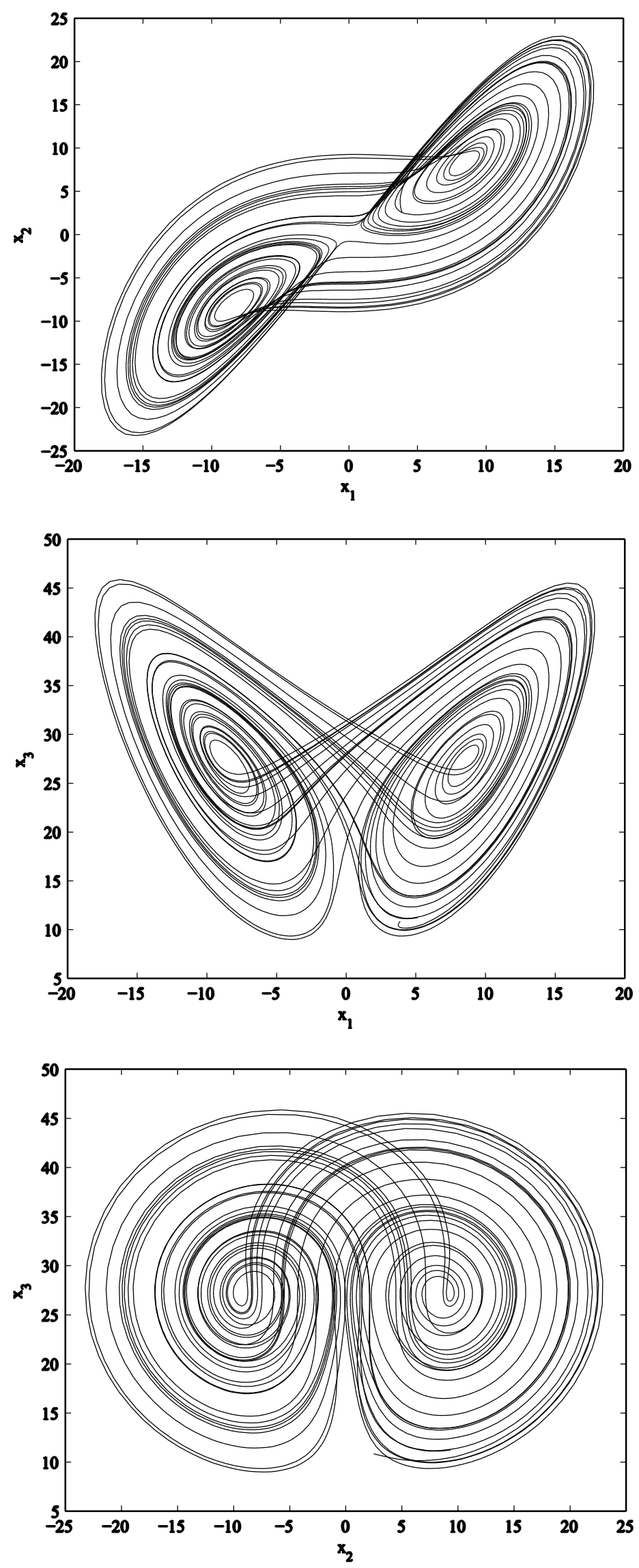


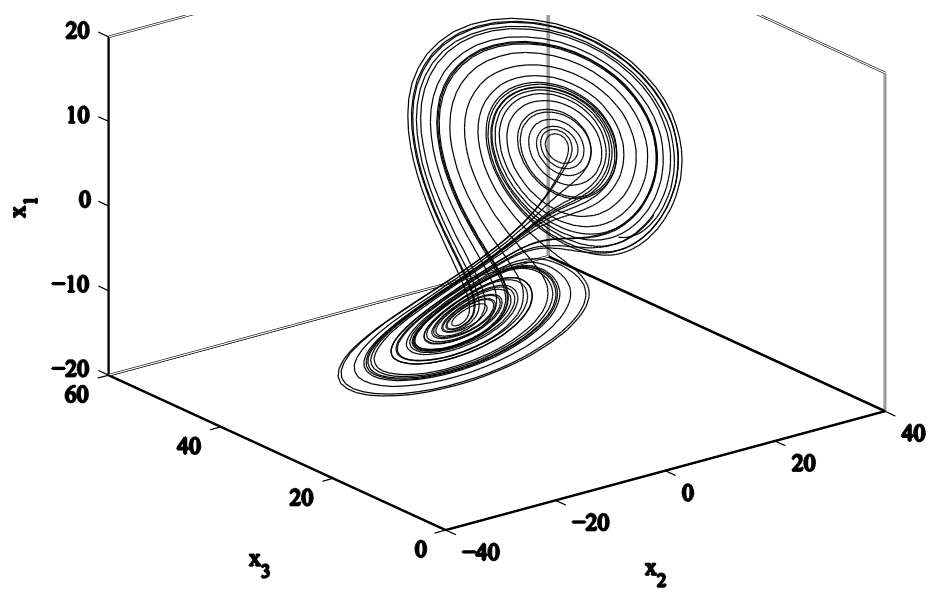

Figure 1. Chaotic behaviour of fractional-order chaotic Lorenz system with the order $\alpha=1.05$.

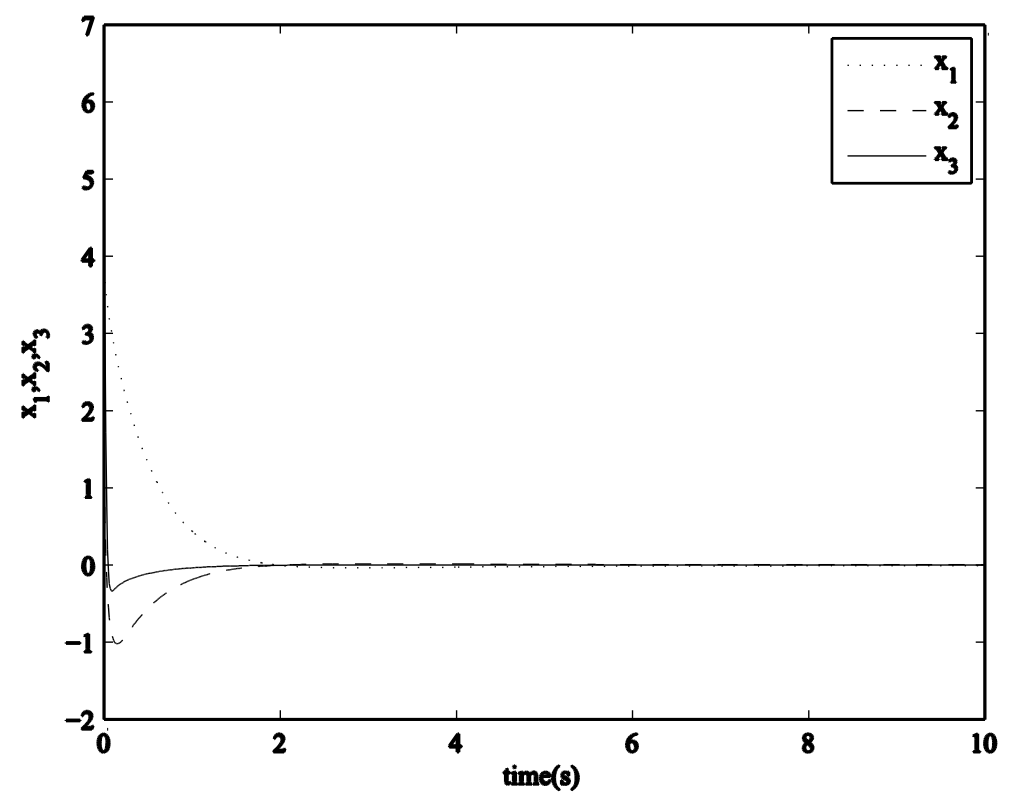

Figure 2. The time response of the states for the controlled Lorenz system with the order $\alpha=1.05$.

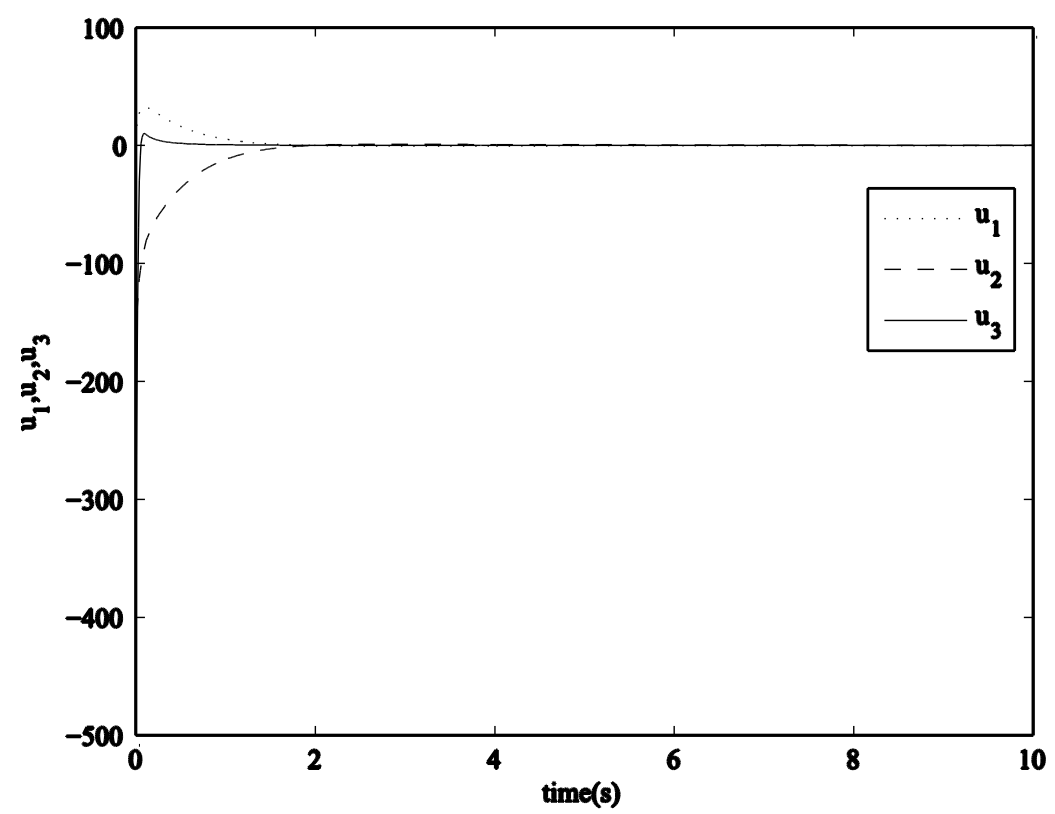

Figure 3. Linear control input for stabilizing fractional-order chaotic Lorenz system with the order $\alpha=1.05$. 


\section{Conclusion}

In this brief, a linear matrix equality condition is proposed to control chaos in fractional-order chaotic systems with order lying in $(1,2)$. The proposed method is economic and effective. The idea and the technique presented in this brief could also be easily extended to synchronize fractional-order chaotic systems.

\section{References}

[1] Andrievskii, B. R. and A. L. Fradkov, "Control of Chaos: Methods and Applications. I. Methods," Automation and Remote Control, 2003, vol. 64, pp. 673-713.

[2] Andrievskii, B. R. and Fradkov, A. L., "Control of chaos: Methods and applications II. Applications," Automation and Remote Control, 2004, pp. 505-533.

[3] Chen, G. R., Controlling chaos and bifurcations in engineering systems, CRC Press, New York, 1999.

[4] Wang, G., Yu, X. and Chen, S., The control, synchronization and application of chaos, The National Defence Industry Press, Beijing, 2001.

[5] DePaula, A. S. and Savi, M. A., "A multiparameter chaos control method based on OGY approach," Chaos Solitons Fractals, 2009, pp. 1376-1390.

[6] Matouk, A. E., "Chaos, feedback control and synchronization of a fractional-order modified Autonomous VanderPol-Duffing circuit," Communications in Nonlinear Science and Numerical Simulation, 2011, pp. 975-986.

[7] Zhong, Q. S., Bao, J. F., Yu, Y. B., et al, "Impulsive control for fractional-order chaotic systems," Chinese Physics Letters, 2008, pp. 2812-2815.

[8] Njah, A. N. and Sunday, O. D., "Generalization on the chaos control of 4-D chaotic systems using recursive backstepping nonlinear controller," Chaos Solitons Fractals, 2009, pp. 2371-2376.

[9] Odibat, Z. M., "Adaptive feedback control and synchronization of non-identical chaotic fractional order systems," Nonlinear Dynamics, 2010, pp. 479-487.

[10] Starrett, J., "Control of chaos by occasional bang-bang," Physical Review E, 2003, pp. 036203.

[11] Dadras, S. and Momeni, H. R., "Control of a fractional-order economical system via sliding mode," Physica A, 2010, pp. 2434-2442.

[12] Ahmad, W. M. and Harb, A. M., "On nonlinear control design for autonomous chaotic systems of integer and fractional orders," Chaos Solitons Fractals, 2003, pp. 693-701.

[13] Nbendjo, B., Salissou, Y. and Woafo, P., "Active control with delay of catastrophic motion and horseshoes chaos in a single well Duffing oscillator," Chaos Solitons Fractals, 2005, pp. 809-816.

[14] Ge, Z. M. and Ou, C. Y., "Chaos in a fractional order modified Duffing system," Chaos solitons Fractals, 2007, pp. 262-291.
[15] Hartley, T. T., Lorenzo, C. F. and Qammer, H. K., "Chaos in a fractional order Chua's system," IEEE Transactions on Circuits and Systems I-Fundamental Theory and Applications, 1995, pp. 485-490.

[16] Li, C. G. and Chen, G. R., "Chaos and hyperchaos in the fractional-order Rössler equations," Physica A, 2004, pp. 55-61.

[17] Li, C. P. and Peng, G. J., "Chaos in Chen's system with a fractional order," Chaos Solitons Fractals, 2004, pp. 443-450.

[18] Grigorenko, I. and Grigorenko, E., "Chaotic dynamics of the fractional lorenz system," Physical Review Letters, 2003, 034101.

[19] Deng, W. H. and Li, C. P., "Chaos synchronization of the fractional Lü system,” Physica A, 2005, pp. 61-72.

[20] Wang, X. Y. and Wang, M. J., "Dynamic analysis of the fractional-order Liu system and its synchronization," Chaos, 2007, pp. 033106.

[21] Chen, W. C., "Nonlinear dynamics and chaos in a fractional-order financial system," Chaos, Solitons Fractals, 2008, pp. 1305-1314.

[22] Petr" $\phi$, I., "Chaos in the fractional-order Volta ${ }^{-} \mathrm{s}$ system: modeling and simulation," Nonlinear dynamics, 2009, pp. 157-170.

[23] Wu, X. J. and Lu, Y., "Generalized projective synchronization of the fractional-order Chen hyperchaotic system," Nonlinear Dynamics, 2009, pp. 25-35.

[24] Wang, T. S. and Wang, X. Y., "Generalized synchronization of fractional order hyperchaotic Lorenz system," Modern Physics Letters B, 2009, pp. 2167-2178.

[25] Yu, Y. G. and Li, H. X., "The synchronization of fractional-order Rössler hyperchaotic systems," Physica A, 2008, pp. 1393-403.

[26] Wajdi, M. A and Reyad, E. K, "Fractional-order dynamical models of love Chaos," Solitons and Fractals, 2007, pp. 1367-1375.

[27] Li, C. G. and Chen, G. R., "Chaos in the fractional order Chen system and its control," Chaos Solitons Fractals, 2004, pp: 549-554.

[28] Wang, X. Y. and He, Y. Y., Wang, M. J, "Chaos control of a fractional order modified coupled dynamos system," Nonlinear Analysis, 2009, pp: 6126-6134.

[29] Zhang, K., Wang, H. and Fang, H., "Feedback control and hybrid projective synchronization of a fractional-order Newton-Leipnik system," Communications in Nonlinear Science and Numerical Simulation, 2011, pp: 317-328.

[30] Matouk, A. E., "Stability conditions, hyperchaos and control in a novel fractional order hyperchaotic system," Physics Letters A, 2009, pp. 2166-2173.

[31] Lu, J. G. and Chen, Y. Q., "Robust stability and stabilization of fractional-order interval systems with the fractional order $\alpha$ : the $0<\alpha<1$ case," IEEE Transactions on Automatic Control, 2010, pp. 152-158.

[32] Matgnon, D., "Proceedings multi conference on computational engineering in systems and application," IMICS, IEEE-SMC, Lille, France, 1996, pp. 963-968. 
[33] Chilali, M., Gahinet, P. and Apkarian, P., "Robust pole placement in LMI regions," IEEE Transactions on Automatic Control, 1999, pp. 2257-2270.

[34] Khargonekar, P. P., Petersen, I. R. and Zhou, K., "Robust stabilization of uncertain linear system: quadratic stabilizability and $\mathrm{h}_{\infty}$ control theory," IEEE Transactions on Automatic Control, 1990, pp. 356-361.
[35] Boyd, S., Ghaoui, L., Feron, E., Balakrishnan V, Linear matrix inequalities in system and control theory, Philadelphia, SIAM, 1994.

[36] El-Khazali, R., Ahmad, W. and Al-Assaf, Y., "Sliding mode control of generalized fractional chaotic systems," International Journal Bifurcation and chaos, 2006, pp. 3113-3125. 